\title{
Sobre un modelo epidemiológico SEI con inmigración
}

\section{On an epidemiological model SEI with immigration}

\author{
Miller Cerón Gómez

\section{Eduardo lbarguen²} \\ Cristian Camilo Espitia ${ }^{3}$ \\ Universidad de Nariño, Colombia \\ Universidad Estadual de Campinas, Brasil.
}

\section{RESUMEN}

Presentamos un modelo matemático tipo SEI que considera la transmisión de una enfermedad por los infectados y los expuestos o latentes. Adicional a esto consideramos que existe un proceso de inmigración en la población. Mostramos que existe un equilibrio endémico y que no existe equilibrio libre de la infección por efecto de la inmigración. Para el equilibrio endémico mostramos la estabilidad global sin restricción en ninguno de los parámetros de existencia.

\section{PALABRAS CLAVE:}

Inmigración, estabilidad global, expuestos en

1 edbargun@udenar.edu.co, Orcid: 0000-00016308-1344 Universidad de Nariño

2 Gómez, millercg@udenar.edu.co, Orcid: 0000-0002-2689-495X, Universidad de Nariño.

3 espitiacristian@gmail.com, Orcid: 0000-00015500-0460. Universidad Estadual de Campinas, Brasil. estado infeccioso, latencia.

\section{ABSTRACT:}

We present a mathematical model type SEI that considers the transmission of a disease by the infected and the exposed or latent. In addition, we consider that there is an immigration process in the population. We show that there is an endemic equilibrium and that there is no free equilibrium of infection due to immigration. For the endemic equilibrium we show global stability without restriction in any of the existence parameters.

\section{KEY WORDS:}

Immigration, global stability, exposed in an infectious state, latency. 


\section{INTRODUCCIÓN}

Los procesos de inmigración de personas siempre han existido, y son parte de nuestra especie, son beneficiosos pues enriquecen las poblaciones culturalmente y genéticamente. Sin embargo cuando se trata de una enfermedad transmisible los procesos de inmigración pueden presentar retos importantes para los sistemas de salud de los países acogedores. En este sentido los modelos matemáticos muestran las posibles dinámicas que podría tener una enfermedad cuando se considera procesos de inmigración. EI modelo que presentamos aquí considera que la transmisión de la enfermedad se produce tanto por personas infectadas como por personas expuestas o personas en estado latente, adicional a esto consideramos que existe un proceso de inmigración en todas las poblaciones a considerar (Susceptibles, expuestos o latentes e infectados). De esta forma, si nuevos casos de infección o estado latente entran en una población harían la erradicación imposible de alcanzar.

Muchos modelos consideran la población expuesta o latente como transmisor de la enfermedad (Cerón Gómez \& Mondragon, 2021; pero ninguno de estos artículos considera los latentes o expuestos como transmisores de la enfermedad como lo hace nuestro artículo.

\section{MODELO SEI}

1

$\bar{\gamma}$ El modelo considera a la población en tres clases: Susceptible, expuesto e infectado, los cuales se denotarán como $S, E$ e $I$, respectivamente. La tasa de reclutamiento por nacimiento es $k_{1}$ para los susceptibles, los parámetros $k_{2}$ y $k_{3}$ corresponden a las tasas de inmigración para las poblaciones de expuestos e infectados, respectivamente. La tasa de incidencia se considera como $\beta S(E+l)$, donde $\beta S E$ corresponde la tasa de incidencia ejercida por la población expuesta o latente y $\beta S /$ corresponde la tasa de incidencia ejercida por la población infectada.
Los expuestos pasan a la población de infectados a una tasa $\gamma E$, el tiempo promedio en que los expuestos permanecen en la población $E$ antes de pasar a la población de infectados es

$$
\frac{1}{\gamma}
$$

Las tasas de mortalidad para las poblaciones de susceptibles, expuestos e infectados son $\mu_{S}, \mu_{E}$ y $\mu_{I}$ respectivamente. De esta manera el modelo a considerar es

$$
\begin{aligned}
& \frac{d S}{d t}=k_{1}-\beta S(E+I)-\mu_{I} S \\
& \frac{d E}{d t}=k_{2}+\beta S(E+I)-\left(\mu_{E}+\gamma\right) E \\
& \frac{d I}{d t}=k_{3}+\gamma E-\mu_{I} I,
\end{aligned}
$$

1.

Todos los parámetros del modelo se consideran positivos. Para estudiar el modelo descrito anteriormente definimos un conjunto

$\Omega=\left\{(S, E, I) \in \mathbf{R}_{+}^{3}: S(t)+E(t)+I(t) \leq \frac{\Lambda}{\delta}\right\}$,

donde

$$
\Lambda=k_{1}+k_{2}+k_{3} \text { y } \delta=\min \left\{\mu_{S}, \mu_{E}, \mu_{7}\right\}
$$

Este conjunto $\Omega$ es positivamente invariante, para una demostración de esto ver (Cerón

Gómez \& Mondragon, 2021)

\section{Puntos de equilibrio.}

Para obtener los puntos de equilibrio debemos solucionar el sistema de ecuaciones siguiente:

$$
\begin{array}{r}
k_{1}-\beta S(E+I)-\mu_{I} S=0 \\
k_{2}+\beta S(E+I)-\left(\mu_{E}+\gamma\right) E=0 \\
k_{3}+\gamma E-\mu_{I} I=0,
\end{array}
$$


Una vez solucionado obtenemos, el punto de equilibrio
2. Usando las ecuaciones de equilibrio, podemos ver que

$$
\begin{aligned}
k_{1} & =\beta S^{*}\left(E^{*}+I^{*}\right)+\mu_{S} S^{*} \\
\mu_{E}+\gamma & =\frac{k_{2}}{E^{*}}+\frac{\beta S^{*}}{E^{*}}\left(E^{*}+I^{*}\right) \\
\mu_{I} & =\frac{k_{3}}{I^{*}}+\gamma \frac{E^{*}}{I^{*}}
\end{aligned}
$$

donde, $a=\beta\left(\mu_{E}+\gamma\right)\left(\mu_{t}+\gamma\right)$

$$
\begin{aligned}
& b=\left(k_{3} \beta+\mu_{3} \mu_{t}\right)\left(\mu_{E}+\gamma\right)-\beta\left(k_{1}+k_{2}\right)\left(\mu_{t}+\gamma\right) \\
& c=-\left(k_{1}+k_{2}\right) k_{3} \beta-\mu_{3} p_{2} k_{2},
\end{aligned}
$$

Cabe notar que no existe el punto de equilibrio libre de la infección puesto que los parámetros son positivos, distintos de cero y $k_{1}, k_{2} y k_{3}$

$$
\left.\frac{d E}{d t}\right|_{E=I=0}=\left.k_{2} \quad y \quad \frac{d I}{d t}\right|_{E=I=0}=k_{3}
$$

esto también implica que no hay número reproductivo básico.

\section{Estabilidad global.}

Teorema: El punto de equilibrio $\left(S^{*}, E^{*}, I^{*}\right)$ es globalmente asintóticamente estable.

Demostración: Sea $L$ la función definida de la forma

$$
\begin{aligned}
L= & S-S^{*}-\ln \frac{S}{S^{*}}+E-E^{*}-\ln \frac{E}{E^{*}}+k\left(I-I^{*}-\ln \frac{I}{I^{*}}\right. \\
& \text { donde } k=\frac{S^{*} I^{*}}{\gamma E^{*}} . \text { Es fácil verificar que esta } \\
& \text { función es de Lyapunov. Ahora, derivando ésta } \\
& \text { función respecto de } t \text { obtenemos que } \\
& \dot{L}=\left(1-\frac{S^{*}}{S}\right) \dot{S}+\left(1-\frac{E^{*}}{E}\right) \dot{E}+k\left(1-\frac{I^{*}}{I}\right) \dot{\bar{I}}
\end{aligned}
$$

y sustituyendo las soluciones del sistema 1), tenemos que

$$
\begin{aligned}
& L=\left(1-\frac{S^{*}}{S}\right)\left(k_{1}-\beta S(E+I)-\mu_{\xi} S\right)+ \\
& \left(1-\frac{E^{*}}{E}\right)\left(k_{2}+\beta S(E+I)-\left(\mu_{E}+\gamma\right) E\right) \\
& +k\left(1-\frac{I^{*}}{I}\right)\left(k_{3}+\gamma E-\mu_{l} l\right)
\end{aligned}
$$

Si sustituimos estas ecuaciones en la ecuación

2. tenemos que

$$
\begin{aligned}
& \mathrm{L}=\left(1-\frac{\mathrm{S}^{*}}{\mathrm{~S}}\right)\left(\beta \mathrm{S}^{*}\left(\mathrm{E}^{*}+\mathrm{I}^{*}\right)+\mu_{\mathrm{S}} \mathrm{S}^{*}-\beta \mathrm{S}(\mathrm{E}+1)-\mu_{5} \mathrm{~S}\right) \\
& +\left(1-\frac{E^{*}}{E}\right)\left(k_{2}+\beta S(E+1)-\frac{k_{2} E}{E^{*}}-\frac{\beta S^{*}}{E^{*}}\left(E^{*}+I^{*}\right) E\right) \\
& +k\left(1-\frac{I^{*}}{l}\right)\left(k_{3}+\eta^{2}-\frac{k_{3} \mid}{\left.\right|^{*}}-\gamma \frac{E^{*} \mid}{r^{*}}\right) .
\end{aligned}
$$

Luego de realizar operaciones, obtenemos

$$
\begin{aligned}
& \dot{L}=-\frac{\left(\mu_{S}+\beta E^{*}\right)}{S}\left(S-S^{*}\right)^{2}-\frac{k_{2}}{E^{*} E}\left(E-E^{*}\right)^{2}-\frac{k k_{3}}{I^{*} I}\left(I-I^{*}\right)^{2} \\
& +\beta S^{*} I^{*}\left(3-\frac{S^{*}}{S}-\frac{S}{S^{*}} \frac{I^{*}}{I^{*}} \frac{E^{*}}{E}-\frac{I}{I^{*}} \frac{E^{*}}{E}\right) .
\end{aligned}
$$

De esta manera, $\dot{L} \quad$ es negativa, puesto que

$$
3-\frac{S^{*}}{S}-\frac{S}{S^{*}} \frac{I}{I^{*}} \frac{E^{*}}{E}-\frac{I^{*}}{I} \frac{E}{E^{*}} \leq 0
$$

Lo cual indica que el punto de equilibrio $\left(S^{*}, E^{*}, I^{*}\right)$ es globalmente asintóticamente estable.

\section{DISCUSIÓN}

EL modelo SEI con inmigración planteado muestra que cuando hay inmigración no existe posibilidad de eliminar la infección, de donde podemos concluir que para mitigar la propagación de una enfermedad lo primero que se debe detener es la inmigración. Por otro lado, el modelo tiene un punto de equilibrio de infección endémico, el cual es globalmente asintóticamente estable.

\section{AGRADECIMIENTOS}

Miller Cerón Gómez agradece a Vicerrectoría de investigaciones-Universidad de Nariño por financiar esta investigación. 


\section{REFERENCIAS BIBLIOGRÁFICAS}

Brauer, F., \& van den Driessche, P. (2001). Models for transmission of disease with immigration of infectives. Mathematical Biosciences, 171(2), 143-154. https://doi.org/10.1016/s00255564(01)00057-8

Cerón Gómez, M., \& Mondragon, E. I. (2021). Global stability analysis for a SEI model with nonlinear incidence rate and asymptomatic infectious state. Applied Mathematics and Computation, 402, 126130. https://doi.org/10.1016/j. amc.2021.126130

Chen, T.-M., Rui, J., Wang, Q.-P., Zhao, Z.-Y., Cui, J.-A., \& Yin, L. (2020). A mathematical model for simulating the phase-based transmissibility of a novel coronavirus. Infectious Diseases of Poverty, 9(1), 24. https://doi.org/10.1186/ s40249-020-00640-3

Gómez, M. C., Mondragon, E. I., \& Molano, P. L. (2020). Global stability analysis for a model with carriers and non-linear incidence rate. Journal of Biological Dynamics, 14(1), 409-420. https://doi. org/10.1080/17513758.2020.1772998

Guo, H., \& Li, M. Y. (2012). Impacts of migration and immigration on disease transmission dynamics in heterogeneous populations. Discrete \& Continuous Dynamical Systems - B, 17(7), 2413. https://doi.org/10.3934/dcdsb.2012.17.2413

Kalajdzievska, D., \& Li, M. Y. (2011). Modeling the effects of carriers on transmission dynamics of infectious diseases. Mathematical Biosciences and Engineering: MBE, 8(3), 711-722. https:// doi.org/10.3934/mbe.2011.8.711

McCluskey, C. C., \& Driessche, P. van den. (2004). Global Analysis of Two Tuberculosis Models. Journal of Dynamics and Differential Equations, 16(1), 139-166. https://doi. org/10.1023/B:JODY.0000041283.66784.3e

Sigdel, R. P., \& McCluskey, C. C. (2014). Global stability for an SEI model of infectious disease with immigration. Applied Mathematics and Computation, 243, 684-689. https://doi.org/10.1016/j. amc.2014.06.020

Wang, L., \& Wang, X. (2012). Influence of temporary migration on the transmission of infectious diseases in a migrants' home village. Journal of Theoretical Biology, 300, 100-109. https:// doi.org/10.1016/j.jtbi.2012.01.004 